THE Astrophysical Journal, 481:447-451, 1997 May 20

(C) 1997. The American Astronomical Society. All rights reserved. Printed in U.S.A.

\title{
HUBBLE SPACE TELESCOPE WFPC2 IMAGES OF EMISSION NEBULOSITY NEAR XZ TAURI
}

\author{
John E. Krist, ${ }^{1}$ Christopher J. Burrows, ${ }^{1,2}$ Karl R. Stapelfeldt, ${ }^{3}$ Gilda E. Ballester, ${ }^{4}$

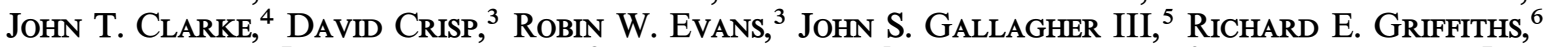 \\ J. Jeff Hester, ${ }^{7}$ Jon A. Holtzman, ${ }^{8}$ John G. Hoessel, ${ }^{5}$ Jeremy R. Mould, $^{9}$ Paul A. Scowen, ${ }^{7}$ \\ John T. Trauger, ${ }^{3}$ Alan M. Watson, ${ }^{8}$ and JAmes A. Westphal ${ }^{10}$ \\ Received 1996 October 2; accepted 1996 December 19
}

\begin{abstract}
$\mathrm{XZ}$ Tauri is a M3 V T Tauri star with a pre-main-sequence binary companion detected by infrared speckle interferometry at a projected separation of 0.3 (about 40 AU). Previous ground-based observations of the system have shown bipolar emission nebulosity, including a possible jet knot. Images obtained with the Wide Field and Planetary Camera 2 on the Hubble Space Telescope show an elongated, filled bubble of emission nebulosity extending over 4 " to the north of the system which contains two or three compact knots. The source of the nebulosity is undetermined. For the first time, the components are separated in visible light, and the southern component (XZ Tau S) dominates the integrated light of the system. The reverse has been found to be true in the near-infrared. Thus we confirm that XZ Tauri belongs to the small class of young binaries with cool infrared companions.
\end{abstract}

Subject headings: ISM: jets and outflows - stars: individual (XZ Tauri) — stars: pre-main-sequence

\section{INTRODUCTION}

$\mathrm{XZ}$ Tauri (HBC 50) is located at an estimated distance of $140 \mathrm{pc}$ (Elias 1978) in the L1551 dark cloud and was once thought to be a single $\mathrm{T}$ Tauri star. It was shown by nearinfrared speckle interferometry to be a 0.3 binary (Haas, Leinert, \& Zinnecker 1990). The southern component (hereafter referred to as XZ Tau S), which we will show to be dominant at visible wavelengths, is an M3 V T Tauri star (Cohen \& Kuhi 1979). The northern one (XZ Tau N) is a possible protostellar object with an estimated color temperature of 1800-1900 K (Haas et al. 1990), and it dominates in the infrared. The system has a significant IRAS excess (Weaver \& Jones 1992), but the small $1.3 \mathrm{~mm}$ flux density of $F_{v}=17 \pm 3 \mathrm{mJy}$ (Osterloh \& Beckwith 1995) indicates a circumstellar mass of only about $10^{-3} M_{\odot}$. Jensen, Mathieu, \& Fuller (1996) demonstrate that this may be due to clearing of the disk by the gravitational influence of the companion. VLA continuum observations by Rodriguez et al. (1994) provide a flux density measurement of $0.23 \mathrm{mJy}$ at $3.6 \mathrm{~cm}$, which they interpret as free-free emission from an ionized wind close to the star-typical of clas-

\footnotetext{
${ }^{1}$ Space Telescope Science Institute, Baltimore, MD 21218; krist@stsci.edu.

${ }_{2}^{2}$ Astrophysics Division, Space Science Department, European Space Agency.

${ }^{3}$ MS 183-900, Jet Propulsion Laboratory, 4800 Oak Grove Drive, Pasadena, CA 91109.

${ }^{4}$ Department of Atmospheric, Oceanic, and Space Sciences, University of Michigan, 2455 Hayward, Ann Arbor, MI 48109.

${ }^{5}$ Department of Astronomy, University of Wisconsin, 475 N. Charter St., Madison, WI 53706.

${ }^{6}$ Department of Physics, Carnegie-Mellon University, Wean Hall, 5000 Forbes Ave., Pittsburgh, PA 15213.

${ }^{7}$ Department of Physics and Astronomy, Arizona State University, Tyler Mall, Tempe, AZ 85287.

${ }^{8}$ Department of Astronomy, New Mexico State University, Box 30001, Department 4500, Las Cruces, NM 88003-8001.

${ }^{9}$ Mount Stromlo and Siding Springs Observatories, Australian National University, Weston Creek Post Office, ACT 2611 Australia.

${ }^{10}$ Division of Geological and Planetary Sciences, MS 170-25 Caltech, Pasadena, CA 91125.
}

sical T Tauri stars that drive outflows. The radio continuum emission must originate from only one of the two XZ Tau components, as this emission was unresolved in a 0"3 VLA beam. Unfortunately, the existing astrometry is not sufficiently accurate to show which star corresponds to the VLA source. Previous and recent centimeter-range measurements are summarized by Wilner, Ho, \& Rodriguez (1996), which agree with those of Rodriguez et al.

Ground-based CCD imaging and spectral measurements (Mundt, Ray, \& Buhrke 1988; Mundt et al. 1990) show a bipolar emission outflow and a nebular condensation at a position angle of about $20^{\circ}$. This orientation is nearly perpendicular to the $100^{\circ}-112^{\circ}$ polarization angle of the system as measured by Bastien (1982). Gledhill \& Scarrott (1989) give a polarization angle of $76^{\circ}$, though they indicate that this is similar to other stars in the periphery of the L1551 cloud. Mundt et al. (1990) traced nebulosity out to $10^{\prime \prime}$ on either side of the stars and reported radial velocities on the order of $40-80 \mathrm{~km} \mathrm{~s}^{-1}$ with the northern component blueshifted.

\section{OBSERVATIONS}

XZ Tau was observed with the Wide Field and Planetary Camera 2 (WFPC2; Burrows 1995) of the Hubble Space Telescope (HST) on 1995 January 5 (HST program 5768). The primary target was HL Tau, which was placed near the center of the Planetary Camera (PC). XZ Tau was located toward the edge of the PC field at the pixel coordinates $(x, y)=(563,682)$. The scale of the PC is 0.0455 pixel $^{-1}$. The observations consist of two $400 \mathrm{~s}$ exposures through filter F675W (WFPC2 $R$ band) at a gain of $7 \mathrm{e}^{-}$per analogto-digital converter unit (ADU), and two $600 \mathrm{~s}$ exposures and one $3 \mathrm{~s}$ exposure in F814W (WFPC2 $I$ band), all at a gain of $14 \mathrm{e}^{-} \mathrm{ADU}^{-1}$. The images were calibrated with the standard HST pipeline, and the duplicate frames were summed, rejecting cosmic rays. In the $\mathrm{F} 675 \mathrm{~W}$ and longexposure F814W frames the star images are saturated and bleed up and down the columns.

The reduced images are shown in the left-hand column of Figure 1 (Plate 16). The small inset image is the unsaturated 
$3 \mathrm{~s}$ F814W image of the binary stars themselves and is the first time the binary has been resolved at visible wavelengths. In the F675W and long F814W frames, a slightly defocused ghost image of the binary is seen about $5^{\prime \prime}$ east of the saturated primary image and is about 8.5 magnitudes fainter. It lies on the line from the CCD center through the stars and is known to be caused by a reflection from the camera's field flattener (Burrows 1995; Krist 1995).

\section{RESULTS}

\subsection{Unsubtracted Images}

As shown in the lower row of Figure 1, the F675W image shows nebulosity forming an elongated bubble extending approximately 4 ".3 (600 AU) to the north at a position angle of $15^{\circ}$, with a maximum width of about $2^{\prime \prime} 8$ (390 AU). Within the bubble is a bright, compact source (knot a), 2".6 from XZ Tau S. The nebula edges appear well defined. The long F814W image shows no evidence for the bubble but does show knot a faintly.

F675W contains the most significant $\mathrm{HH}$ object emission lines ([S II $] 6717,6731 \AA, \mathrm{H} \alpha 6563 \AA$, and [O I $] 6300 \AA$ ). F814W contains weaker lines, including [Fe II] $8617 \AA$. It is therefore possible to distinguish reflection nebulosity, which tends to have neutral to red colors, from emission nebulae, which are apparently very blue in these two passbands. The absence of the bubble in the F814W image $(I-R>2)$ indicates that it is unlikely to be reflection nebulosity. A photoionized nebula is ruled out by the fact that XZ Tau S is a late-type star and thus is a negligible source of ionizing photons. The emission-line spectrum of a Herbig-Haro object can reproduce the broadband colors of the bubble. Therefore the bubble is probably an emission nebula tracing a wind from the XZ Tau system. This hypothesis is supported by the ground-based long-slit spectroscopy and astrometry in Mundt et al. (1990), which show an emissionline spectrum near this position and outflowing material at this position angle.

\subsection{PSF Subtraction}

The bubble can be traced to within 1.5 of the stars, where the point spread function (PSF) features begin to dominate. The PSFs must be subtracted in order to see the nebulosity within this radius. XZ Tau presents an especially difficult case since its components are separated by only a few pixels and are far from the chip center where reference PSFs are available, nebulosity is within the wings of the PSFs, and fluxes must be estimated from saturated images (in the case of $\mathrm{F} 675 \mathrm{~W}$ ). The techniques used for the PSF subtractions are given here in some detail because they can and are being employed in many other HST observations of circumstellar and circumnuclear matter near bright unresolved sources.

The diffracted and scattered light in the wings of the PSF core is not uniform. It is characterized by streaks radiating from the star, superposed on a background that is itself modulated in roughly concentric rings. These features change in intensity and position depending in rough order on the filter used, the field position of the object, the timevariable focus of the telescope, the object color (for broad bandpasses), details of the pointing accuracy of the telescope, exposure time (for exposures less than about a second), and the contamination state of the instrument for blue passbands $(\lambda<5000 \AA)$. Ideally, one should obtain a series of properly exposed stellar PSFs through the same filter, at a variety of positions within about 30 pixels of the objects and with a similar target color and less critically at a similar epoch (within a few days for blue passbands and a few weeks otherwise). Even then, experience shows that the result is an rms noise level of about $15 \%$ of the local target PSF level. A nonoptimal PSF can easily lead to RMS errors at the $30 \%-50 \%$ level.

An extensive review of archived HST images showed no similarly saturated PSFs near the XZ Tau position. The closest useful PSFs were near the center of the PC, about $11^{\prime \prime}$ away. These were images of the naked T Tauri stars SAO 76411A in F675W (HST program 5204) and HDE 283572 in F814W (HST program 6223). These two stars were subtracted by each other, as well as by other stars, and revealed no evidence for circumstellar material.

An alternative to using an observed PSF is to create a simulated one using Tiny Tim (Krist 1996). A model can be generated for any given position, filter, object color, and spatial sampling. However, because of uncertainties in the optical parameters of the telescope (i.e., focus, field dependent aberrations, and scattering properties), significant mismatches between a model PSF and an observed one are expected. For our purposes, the most significant model errors occur between 0.4 and 3 " from the star, where the lack of large angle scattering in the Tiny Tim models results in undersubtraction of the wings. We examined subtractions using both the observed PSFs and the Tiny Tim models to ensure that real structures are identified instead of PSF artifacts.

Given that the brighter component, XZ Tau S, is an M3 star, models for each filter were computed using Tiny Tim V4.1 for the reddest object color standard to the software $(B-V=1.59)$. However, a comparison by blinking the simulation and the long F814W XZ Tau image showed that the scattering streaks in the PSF halo of XZ Tau S were farther away from the star than in the model. Changing the simulation parameters to include a reasonable amount of defocus did not significantly improve the agreement.

We decided that a mismatch in object color was the problem. The extinction to XZ Tau is about $A_{V} \approx 3$ (Strom et al. 1989), which makes XZ Tau S somewhat redder than the color used in the models. Tiny Tim creates a PSF for a given filter and object color using a weighted sum of individual monochromatic PSFs generated at wavelengths sampling the filter's bandpass. Because diffraction structures in a PSF expand with increasing wavelength, greater weighting toward the red end of a filter will force the streaks outward, as desired. To determine reasonable weights, the spectrum of a $3360 \mathrm{~K}$ blackbody, corresponding to an M3 star, was reddened by $E(B-V)=1\left(A_{V} \approx 3\right)$ and multiplied by the filter and camera throughputs at the sample wavelengths. The default weights used by Tiny Tim were manually altered to these values, and the resulting PSF was a good match in terms of streak positions.

The positions and intensities of the two stars in the $3 \mathrm{~s}$ F814W exposure were determined by the following procedure. A simulated F814W PSF, subsampled by a factor of 5 in each dimension, was generated using the revised weights. Since both stars have similar $R-I$ colors, the same PSF can be used for both without concern for color differences. An iterative fitting routine was used to create a synthetic image of the two stars based on this PSF. In each iteration, the PSFs were shifted using interpolation to the locations of the stars, intensity scaled, and added together. 
This image was then rebinned to normal sampling and convolved with a kernel representing pixel-level scattering, as described in Krist (1996). The routine minimized the $\chi^{2}$ difference between the real and synthetic images. The wings of the PSFs were in the noise in the short exposure, so the previously mentioned underestimation of flux there in the model PSFs was not important.

There were some noticeable residuals within $00^{\prime \prime} 2$ of the PSF cores, skewed toward one side of the stars. Since both stars showed the same pattern, these were due to incorrect aberrations in the models rather than nebulosity. The Planetary Camera has small field-dependent focus, coma, and astigmatism terms that are not included in Tiny Tim models by default. To obtain a better model, we used phase retrieval software (Krist \& Burrows 1995) to measure the aberrations by fitting the $3 \mathrm{~s}$ F814W image of XZ Tau S (XZ Tau $\mathrm{N}$ was masked out). Another Tiny Tim model was generated using the retrieved values, which effectively removed this residual pattern.

The fluxes derived from the $3 \mathrm{~s}$ F814W model PSF normalizations are 52980 and $10082 \mathrm{e}^{-} \mathrm{s}^{-1}$ for XZ Tau S and $\mathrm{N}$, respectively, which correspond to instrumental magnitudes of WFPC2 $I=11.99$ and 13.79 with estimated errors of $3 \%$. These values compare well with the combined flux of $63254 \mathrm{e}^{-} \mathrm{s}^{-1}$ (WFPC2 $I=11.79$ ), measured using a $1^{\prime \prime}$ radius aperture. The integrated magnitude is also in excellent agreement with ground-based measurements (Strom et al. 1989).

We estimate that the positions are accurate to within 0.1 pixel (0"005). One cannot expect better accuracy because of uncertainties in the PSF. The measured separation, corrected for geometric distortion, is $00^{\prime \prime} 307 \pm 00^{\prime \prime} 009$. This is in agreement with the values determined from infrared speckle interferometry by Haas et al. (1990) $(0.30 \pm 0$ " .02$)$ and Ghez et al. (1995) $\left(00^{\prime \prime} 31 \pm 0.01\right.$ in 1991 and $0^{\prime \prime} .296 \pm 0$ 0 ${ }^{\prime \prime} 002$ in 1994). The measured position angle is $147.8 \pm 0.25$ based on the WFPC2 PC1 orientation given in the image headers. Haas et al. measured a position angle of $154^{\circ} \pm 3^{\circ}$ in 1989 , and Ghez et al. (1995) determined PA $=151^{\circ} \pm 2^{\circ}$ in 1991 and $147.0 \pm 0.4$ in 1994 (18 days before our observations). These measurements may suggest relative motion between the two stars. The difference between our measurement and what Haas et al. found $6 \mathrm{yr}$ earlier is about $6^{\circ}$. For a $0.5 M_{\odot}$ star, the orbit period at $50 \mathrm{AU}$ radius is $500 \mathrm{yr}$, which corresponds to a circular orbit motion of 1.4 per $\mathrm{yr}-$ consistent with our observations. However, the error bars on all the values are too large to make any accurate assessment of orbital motions.

The synthetic image based on the $3 \mathrm{~s}$ F814W frame, scaled by the exposure time, was subtracted from the $600 \mathrm{~s}$ image. The F675W subtraction was more complicated because the saturated images prevented direct measurement of the fluxes of the two components, and there was a shift of a few pixels between the F675W and F814W exposures. Automated PSF fitting, as was done for the $3 \mathrm{~s} \mathrm{F814W}$ image, could not be used for F675W because of nebulosity and saturation.

By trial and error, $E(B-V)=1$ reddened model F675W PSFs of various normalizations were added together with the measured separation. Each synthetic image was aligned by matching the diffraction spikes of the stars, and the normalizations were set by comparing cross-sectional plots and visually examining the subtractions. Because of mismatches in the diffraction patterns caused by differences in position, focus, and modeling errors, the spiders cannot be used to establish normalizations. Because of the lack of large angle scattering in the models, there is a residual halo of unsubtracted light out to about 3 " when the normalizations are optimized for the region near the core.

We used F675W models with both the default Tiny Tim aberrations and those from the F $814 \mathrm{~W}$ phase retrieval. The PSF generated using the revised aberrations was not a good match to the observed image, based on asymmetries seen in the diffraction rings. This is probably because the coma varies with each filter in WFPC2. The default aberration model was used instead, since phase retrieval cannot be performed well on highly saturated images. The derived F675W fluxes are 25768 and $5041 \mathrm{e}^{-} \mathrm{s}^{-1}$ for XZ Tau S and $\mathrm{N}$, respectively (WFPC2 $R=13.16$ and 14.93). The estimated errors are about $15 \%$ for each star.

The normalization procedure was tested on the long F814W exposure. The F814W model PSFs were separately normalized and placed at the measured separation, as described for the F675W images. This provided photometry independent of that measured using the short F814W image. The measured fluxes were 56417 and $10375 \mathrm{e}^{-} \mathrm{s}^{-1}$ for XZ Tau S and N, respectively (WFPC2 $I=11.92$ and 13.76), with estimated errors of $10 \%$.

An additional check on the derived fluxes was aperture photometry of the ghost images, which agreed with the PSF normalizations to within $20 \%$. The ghost-to-image flux ratio was defined by the F814W XZ Tau S measurements.

As with the models, the observed SAO 76411A (F675W) and HDE 283572 (F814W) PSFs were duplicated, shifted to the measured separation, and intensity scaled. These have the same average level of scattered light in the wings as $\mathrm{XZ}$ Tau, which is an advantage over the models. However, since the PSFs are from different field positions than XZ Tau and are not the same color (both are $G$ types), the diffraction structures near the core are very different. This problem also extends to the wings, with mismatches in the streak patterns, especially in F814W. The noise in the wings made it difficult to determine the correct normalizations for $\mathrm{XZ}$ Tau S, resulting in estimated errors of $25 \%$. The derived fluxes are $65299(\mathrm{~F} 814 \mathrm{~W})$ and $34722(\mathrm{~F} 675 \mathrm{~W}) \mathrm{e}^{-} \mathrm{s}^{-1}$ (WFPC2 $I=1.76$ and WFPC2 $R=12.83$ ). It was impossible to determine reasonably accurate normalizations for subtracting $\mathrm{XZ}$ Tau $\mathrm{N}$, so the $\mathrm{XZ}$ Tau $\mathrm{S}$ values were multiplied by the flux ratios derived from the model PSF subtractions.

\subsection{PSF Subtraction Results}

In the end, subtraction of a model PSF provided a cleaner F675W image of the nebulosity, which is significantly brighter than the residual halo. The F814Wobserved PSF subtraction shows that there is no obvious reflection nebulosity. Photometry results for the two stars are summarized in Table 1.

In the PSF-subtracted F675W image the bubble can be seen to within 0.5 of the stars, where it becomes lost in the subtraction residuals. There are hints of a possible counternebula $1^{\prime \prime}$ to the south of the system. The nebula appears to converge toward XZ Tau N, though it still may be about 1".5 wide at the star. The average surface brightness of the nebula is approximately $R=20.5 \mathrm{mag} \operatorname{arcsec}^{-2}$ at $2^{\prime \prime}$ from the stars. There is considerable mottling or filamentary structure. Between 1" and 4" from the stars, the average surface brightness decreases roughly linearly (excluding the 
TABLE 1

XZ TAu S(N) Photometry

\begin{tabular}{llll}
\hline \hline \multicolumn{1}{c}{ Flux } & \multicolumn{1}{c}{ F675W } & \multicolumn{1}{c}{ F814W } & \multicolumn{1}{c}{$R-I$} \\
\hline Short exposure .......... & N/A & $11.99(13.79)$ & N/A \\
Model subtraction ....... & $13.16(14.93)$ & $11.92(13.76)$ & $1.24(1.17)$ \\
Star subtraction ........ & $12.83($ N/A) & $11.76($ N/A) & 1.07 (N/A) \\
Ghosts ................. & $12.95(14.77)$ & $11.99^{\mathrm{a}}(13.67)$ & $0.96(1.10)$ \\
\hline
\end{tabular}

${ }^{a}$ Ghost flux set to short exposure flux for F814W XZ Tau S.

knots). The far end of the bubble appears to blend into the background, perhaps indicating that it is open ended.

Between knot a and the stars is a similarly compact, brighter knot, $b$, which is $1{ }^{\prime \prime} 1$ from XZ Tau $S$. On the opposite side of the stars is knot c, at a distance of 1".3, which is considerably fainter than knot a or b. It may be a condensation in the bubble or an extincted jet knot. The approximate $R$ magnitudes of the knots are 22.0, 21.3, and 22.5 for knots $a, b$, and c, respectively $( \pm 0.5 \mathrm{mag})$. These values are similar to those measured in the jet of the nearby (to XZ Tau) protostar HH 30 (Burrows et al. 1996). The FWHM of the knots is about 0.14 (20 AU), also similar to $\mathrm{HH}$ 30. This is wider than an unresolved source, thus they are not stars, and no other stellar sources were reported in the IR interferometry observations. No other knots are visible within $11 . .5$ of the system.

A line drawn through knots $a$ and $b$ passes about 0 ".2 east of XZ Tau S, on the side opposite XZ Tau N, and also passes through knot c. The position angle of this line is $9.9 \pm 0.3$, and it is offset eastward of the center line of the nebula by about 0.25 . The possible knot detected by Mundt et al. (1990), which they designated XZ-B,was located 10" from XZ Tau at a position angle of $20^{\circ}$. Their knot was not seen in our observations, either because of its low surface brightness or because its proper motion had carried it outside our field of view.

The source of the outflow cannot be clearly determined from the knot alignment or the bubble orientation in these images. A jet from XZ Tau $\mathrm{S}$ would have to be bent or precessing by about $3^{\circ}$ to trace through knots a and $b$. For $\mathrm{XZ}$ Tau N, the angle would be about $15^{\circ}$. The possible knot seen by Mundt et al. would lie within this range but would increase the flow deflection angle for XZ Tau S to about $6^{\circ}$. Since XZ Tau N is presumably the younger and more active component (based on the derived temperature), it would seem the likely source, but its angle is exceptionally large. Of course, all this assumes that what are observed are actually jet knots. Proper motion measurements for the bubble and knots would greatly clarify which star is the source of the outflow.

Knot $a$ is faintly seen in $F 814 W$, which contains a number of less prominent emission lines. Its estimated $I$ magnitude is $24.5 \pm 1.0$. The brighter knot $b$ is not detected, though it lies in a region of significant residuals, as does knot c. There is some residual signal in the region of the nebula in the F814W subtractions, but this is at the level of the residuals in other parts of the PSF wings. These measurements are consistent with the knots being continuumfree emission-line nebulae.

\section{DISCUSSION}

\subsection{The Nature of the IR Companion}

With separate optical photometry of the two binary components, it becomes possible to constrain the spectral type of each source. For XZ Tau S, a $3360 \mathrm{~K}$ blackbody reddened by $E(B-V)=1$ predicts an $R-I$ color $(1.18 \mathrm{mag})$ very close to the observed value of $1.24 \mathrm{mag}$. More interesting are the photometry results for XZ Tau N. Haas et al. assigned this source a color temperature of $1900 \mathrm{~K}$ based on infrared photometry between 1 and $3 \mu \mathrm{m}$. They were unable to derive an extinction toward $\mathrm{XZ}$ Tau $\mathrm{N}$ since they lacked optical photometry. As shown in Figure 2, the measured $I$-band flux for XZ Tau N lies directly on the $1900 \mathrm{~K}$ blackbody curve extrapolated from the near-IR photometry. This strongly suggests that the extinction toward $\mathrm{XZ}$ Tau $\mathrm{N}$ is small, that the low color temperature is real, and thus that XZ Tau N may be a substellar or protostellar object. However, our $R-I$ color for $\mathrm{XZ}$ Tau N $(1.17 \pm 0.20)$ is better matched to a higher color temperature like that of $\mathrm{XZ}$ Tau S. A direct determination of the spectral type of XZ Tau $\mathrm{N}$ is needed to verify the unusually low color temperature of this object.

\subsection{Origin of the Bubble}

The paradigm for Herbig-Haro flows includes a collimated jet and a bow shock where the jet flow impacts surrounding ambient material. It is tempting to apply this model to the bubble near XZ Tauri. In this view, knots a and $b$ would define a jet axis that nearly bisects the bubble; the bubble itself would be identified as the bow shock. There are two significant difficulties with this model for $\mathrm{XZ}$ Tau. First, the superposition of the jet knots against the bubble and the bubble's small angular extent from the star suggest that such a flow would be significantly foreshortened and thus viewed from near the flow axis. However, ground-based measurements show that the radial velocity in this region is much too small to be consistent with a typical Herbig-Haro flow directed nearly toward the observer. Second, the nebula appears to be a filled bubble, showing no limb brightening, which would be expected for a hollow shell. The edges should be at least a factor of 2 greater in surface brightness than the interior if the emission were confined to a thin surface such as a typical bow shock.

Mundt et al. (1990) estimate that the XZ Tau outflow is inclined $\approx 20^{\circ}$ from the plane of the sky, based on the measured radial velocity of $\approx 100 \mathrm{~km} \mathrm{~s}^{-1}$ and an assumed jet

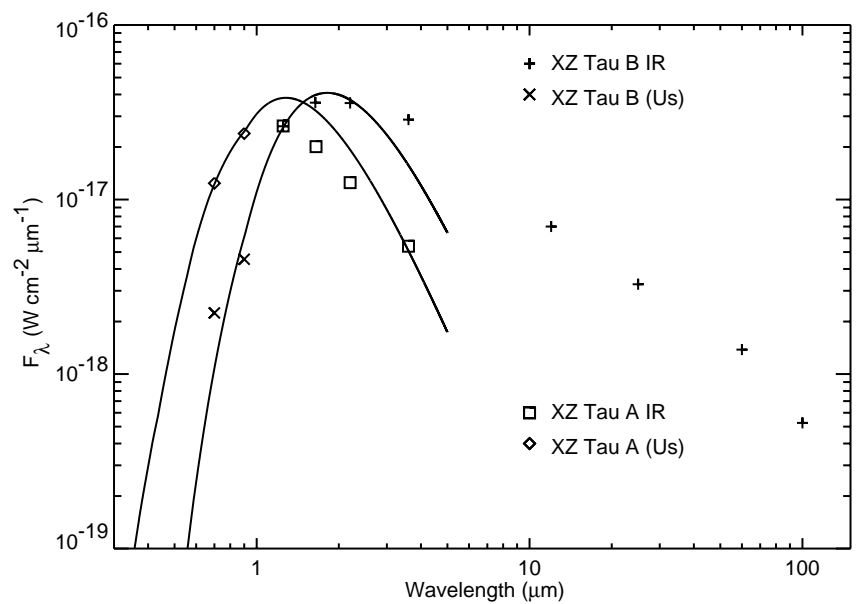

Fig. 2.-XZ Tau $\mathrm{S}$ and $\mathrm{N}$ photometry. The $R$ - and $I$ - band fluxes from this study, $J$-, $H$-, $K$-, and $L$-band fluxes from Haas et al. (1990), and IRAS 60-100 $\mu \mathrm{m}$ fluxes from the point source catalog are shown. Blackbody curves with $E(B-V)=1$ extinction are plotted at $1900 \mathrm{~K}$ and $3360 \mathrm{~K}$. 
velocity of $400 \mathrm{~km} \mathrm{~s}^{-1}$. This inclination and flow speed imply a very young dynamical age for the $\mathrm{HH}$ objects near XZ Tauri. Knot a would have a dynamical age of just $6 \mathrm{yr}$, and the entire bubble a dynamical age of only $10 \mathrm{yr}$. The knots appear very compact and do not seem to have been deformed by interaction with the surrounding medium. Given that the nebula is so sharply defined and given its apparent youth, it probably originated in a recent outburst of accretion and outflow after a period of dormancy. In this view, the bubble may represent an early stage in the evolution of a jet-bow shock system. The Mundt et al. (1990) observations, with better sensitivity to low surface brightness features, detected more distant nebulosity that would be related to earlier outbursts from the system. The lack of limb brightening remains a problem for the bow shock model. Possible solutions to this are a spatially resolved postshock cooling zone comparable in thickness to the bubble itself or a complex series of internal shocks within the bubble. An attractive possibility is that a preexisting outflow cavity is shocked and heated by the reemergence of a high-velocity jet. The denser surrounding envelope is not heated enough to ionize. In this view, we would expect the bubble to grow in size as the jet propagates away from the star. It will be possible to simply distinguish these models by images in emission lines ( $\mathrm{H} \alpha$ and $[\mathrm{S} \mathrm{II}]$ ) at several epochs.

At first, it is perplexing that the flow appears monopolar rather than bipolar. However a small amount of extra extinction (2 mag) would imply that knot $\mathrm{c}$ is intrinsically only 1 mag brighter than knot $b$, while it would hide the counternebula (which is only present at the few DN level) almost entirely in the noise. Such extinction could be caused by the outer regions of a flared circumbinary disk or surrounding envelope.

\section{CONCLUSIONS}

HST observations of XZ Tau have enabled us to clearly separate the visible light from the binary components and measure their individual magnitudes, colors, and separation. In addition, we see an apparent filled bubble of emission nebulosity that demands explanation. The bubble contains at least two knots of emission nebulosity that resemble in brightness and size the knots detected in the jets from other YSO systems. They are not well aligned with either component of the binary, so if there is a jet, it must either change direction at the source with time (perhaps precess) or bend in response to density gradients in the external medium.

HST has perhaps observed a YSO jet that started within the last 10 years or so. It would then be in the process of forming a new terminal bow shock, or else it has heated and ionized tenuous material in the outflow cavity. Future observations of the system will help us to determine the source of the outflow and distinguish these possibilities.

This work was supported by the WFPC2 Investigation Definition Team under a grant from the National Aeronautics and Space Administration.
Bastien, P. 1982, A\&AS, 48, 153

Burrows, C. J., ed. 1995, Hubble Space Telescope Wide Field and Planetary Camera 2 Instrument Handbook. Version 3.0 (Baltimore: STScI)

Burrows, C. J., et al. 1996, ApJ, 473, 437

Cohen, M., \& Kuhi, L. V. 1979, ApJS, 41, 743

Elias, J. H. 1978, ApJ, 224, 857

Ghez, A. M., Weinberger, A. J., Neugebauer, G., Matthews, K., \& McCarthy, D. W., Jr. 1995, AJ, 110, 753

Gledhill, T. M., \& Scarrott, S. M. 1989, MNRAS, 236, 139

Haas, G. M., Leinert, C., \& Zinnecker, H. 1990, A\&A, 230, L1

Jensen, E. L. N., Mathieu, R. D., \& Fuller, G. A. 1996, ApJ, 458, 312

Krist, J. E. 1995, in Calibrating Hubble Space Telescope: Post Servicing Mission (Baltimore: STScI), 311

\section{REFERENCES}

Krist, J. E. 1996, Tiny Tim User's Manual V4.1

Krist, J. E., \& Burrows, C. J. 1995, Appl. Opt., 34, 4951

Mundt, R., Buhrke, T., Solf, J., Ray, T. P., \& Raga, A. C. 1990, A\&A, 232, 37

Mundt, R., Ray, T. P., \& Buhrke, T. 1988, ApJ, 333, L39

Osterloh, M., \& Beckwith, S. V. W. 1995, ApJ, 439, 288

Rodriguez, L. F., Canto, J., Torrelles, J. M., Gomez, J. F., Anglada, G., \& Ho, P. T. P. 1994, ApJ, 427, L103

Strom, K. M., Strom, S. E., Edwards, S., Cabrit, S., \& Skrutskie, M. F. 1989, AJ, 97,1451

Weaver, W. B., \& Jones, G. 1992, ApJS, 78, 239

Wilner, D. J., Ho, P. T. P., \& Rodriguez, L. F. 1996, ApJ, 470, L117 


\section{F814W}
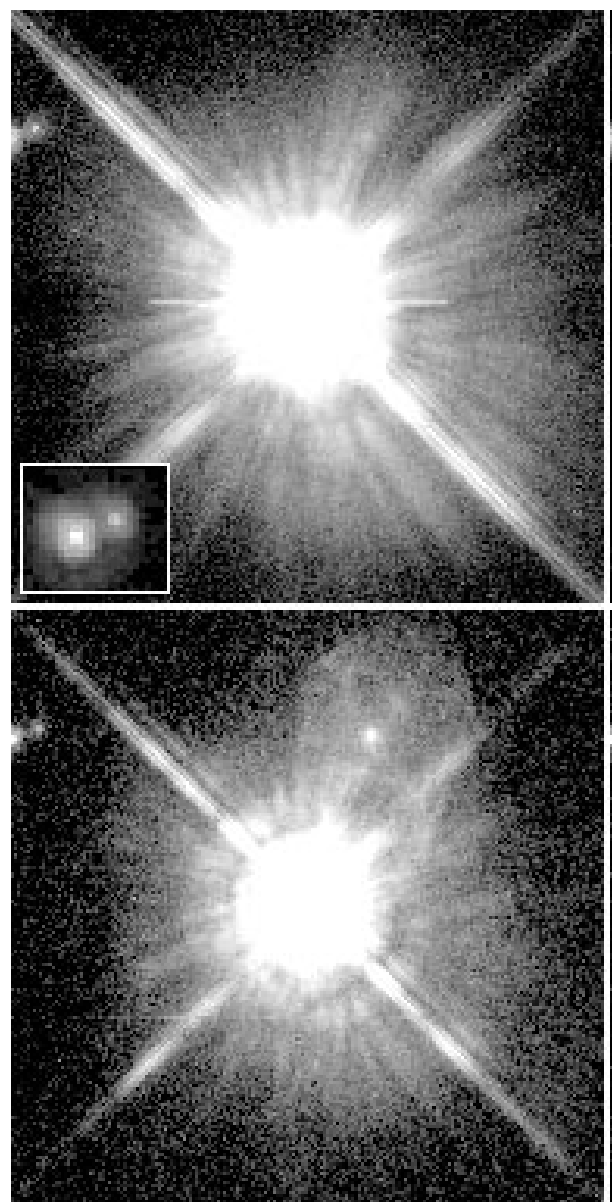

F675W
F814W - HDE 283572
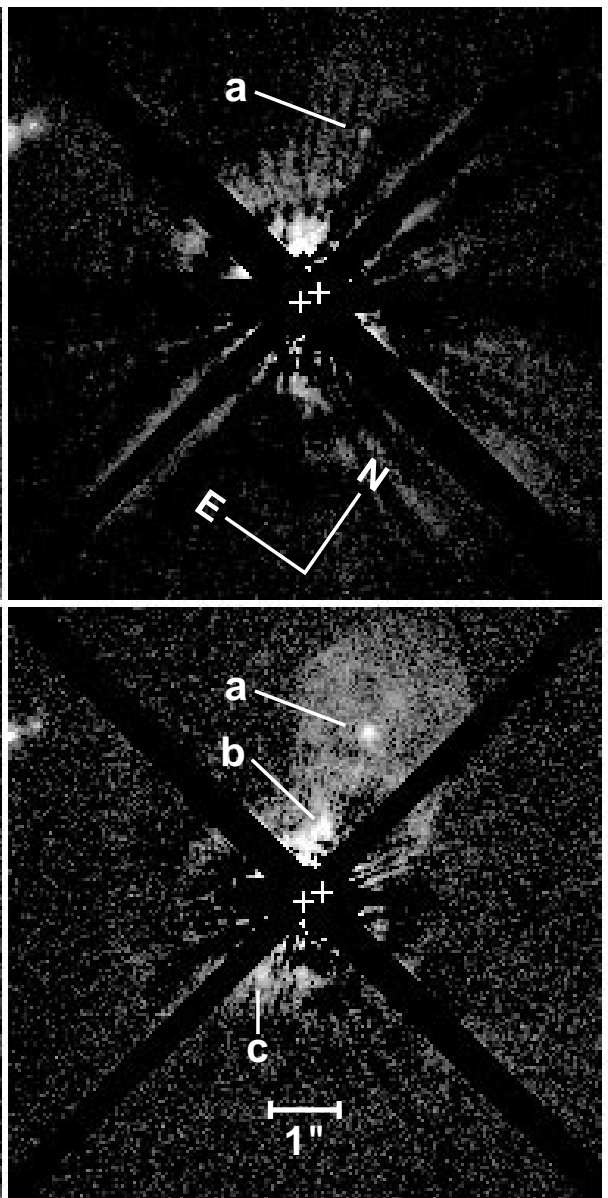

F675W - SAO 76411A
F814W - Model PSF
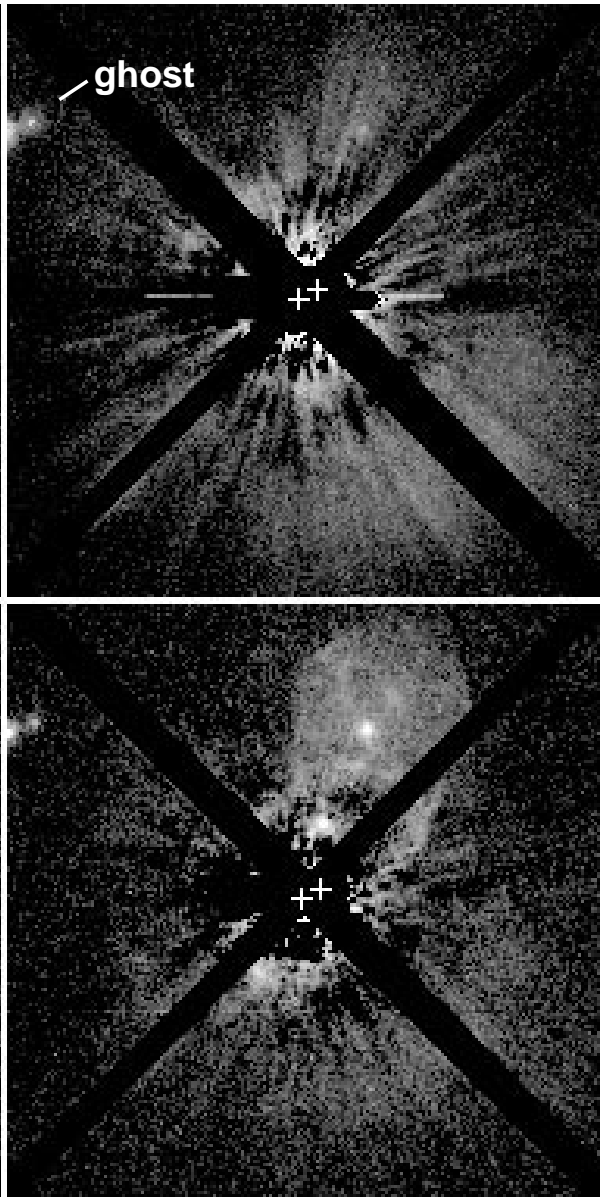

F675W - Model PSF

FIG. 1.-HST WFPC2 Planetary Camera $\left(0^{\prime \prime} 0455\right.$ pixel $^{-1}$ ) images of XZ Tau in filters F814W (WFPC2 I) and F675W (WFPC2 R). Left: Unsubtracted images, with the short F814W image magnified by a factor of 2 in the inset. Middle: PSF subtractions using the indicated observed stars. Right: Subtractions using Tiny Tim PSF models. The crosses mark the positions of XZ Tau S and N. Knots a, b, and c are identified, as is the ghost image of XZ Tau. The images are logarithmically scaled between 0.5 and $25 \mathrm{DN}$, except for the inset, which is logarithmically scaled between 1 and $1196 \mathrm{DN}$. Residuals from diffraction spikes and saturated columns have been masked in the subtracted images.

KRIST et al. (see 481, 447) 